\title{
Renal Cell Carcinoma with Skin metastasis: A Case Report and Literature Review
}

\author{
Asim Ahmad ${ }^{1}$, Kevin $\mathrm{Wu}^{2}$ \& Winston $\mathrm{Tan}^{3}$ \\ ${ }^{1}$ Internal Medicine, Mayo Clinic, Jacksonville, United States \\ 2 Pathology, Mayo Clinic, Jacksonville, United States \\ ${ }^{3}$ Hematology Oncolocgy, Mayo Clinic, Jacksonville, United States \\ Correspondence: Dr. Asim Ahmad, Internal Medicine, Mayo Clinic, Jacksonville, United States. Tel: \\ 1-904-953-2000. E-mail: ahmad.asim@mayo.edu
}

Received: November 26, 2012 Accepted: December 11, 2012 Online Published: January 9, 2013

doi:10.5539/cco.v2n1p80 URL: http://dx.doi.org/10.5539/cco.v2n1p80

\begin{abstract}
Background: Renal cell carcinoma (RCC) comprises $80 \%$ of primary renal cancers in the USA. Most cases are discovered incidentally during imaging for other reasons. The majority of patients are asymptomatic upon diagnosis, yet $25 \%$ have advanced disease. The most common locations for metastasis include liver, lymph nodes, bone, and lungs. RCC presents as skin metastasis in $6 \%$ of cases. Here, we present an even rarer occurrence: of recurrent RCC presenting with cutaneous metastases following nephrectomy and immunotherapy. Case Report: A 75 year-old white male presented to his physician for a yearly exam. Routine urinalysis revealed microcytic hematuria. Ultrasound demonstrated a solid mass in the upper pole of the right kidney. CT scan of the chest revealed pulmonary nodules. Patient underwent a right radical cyto-reductive nephrectomy, biopsy of mesenteric mass, and left thoracotomy with biopsy. Final pathology revealed grade 4 RCC, clear-cell type. Patient had treatment with interferon Alpha and then interleukin-2. As third line treatment, patient was switched to bevacizumab. While on treatment, patient presented a lesion on his left back and diffuse bone pain. During physical exam, a $2 \times 2.5 \mathrm{~cm}$ firm, immobile, subcutaneous nodule was noted. Pathological analysis revealed a tumor consistent with metastatic RCC. MRI of the brain revealed calvarial lesions with some break into the scalp soft tissue and into the epidural space. Erlotinib was added to bevacizumab. Conclusion: This case helps highlight the ubiquity of RCC metastasis. The most common locations for metastasis include lung, liver, local lymph nodes, bone, and brain. In RCC, skin lesions have been described in the literature more specifically as pustules, painful and painless nodules, and macular lesions. The differential diagnosis of a skin lesion include non-specific drug reactions, opportunistic skin infections, and metastatic disease. The definite diagnosis of metastasis is made through tissue biopsy.
\end{abstract}

Keywords: renal cell carcinoma, metastasis, skin, review

\section{Introduction}

We present, a 75 year-old man with renal cell carcinoma (RCC) that had biopsy proven metastasis. This case is unique in that this patient's disease metastasized to the skin. There have been very few cases of such reported in the literature, especially in regards to patients that are status post- nephrectomy.

\section{Case Report}

75 year-old white male presented to his physician for a yearly exam. Routine urinalysis revealed microcytic hematuria. Patient's genitourinary review of symptoms was unremarkable and a complete physical exam revealed no abnormalities. Urinalysis was repeated and remained unchanged. His performance status Eastern Cooperative Group was 0.

Ultrasound demonstrated a solid mass in the upper pole of the right kidney. CT scan revealed pulmonary nodules. MRI demonstrated a large, centrally necrotic mass in the right kidney with extension into the right renal vein (Figure 1). Octreo-scan showed increased uptake in the kidney, consistent with imaging findings. 


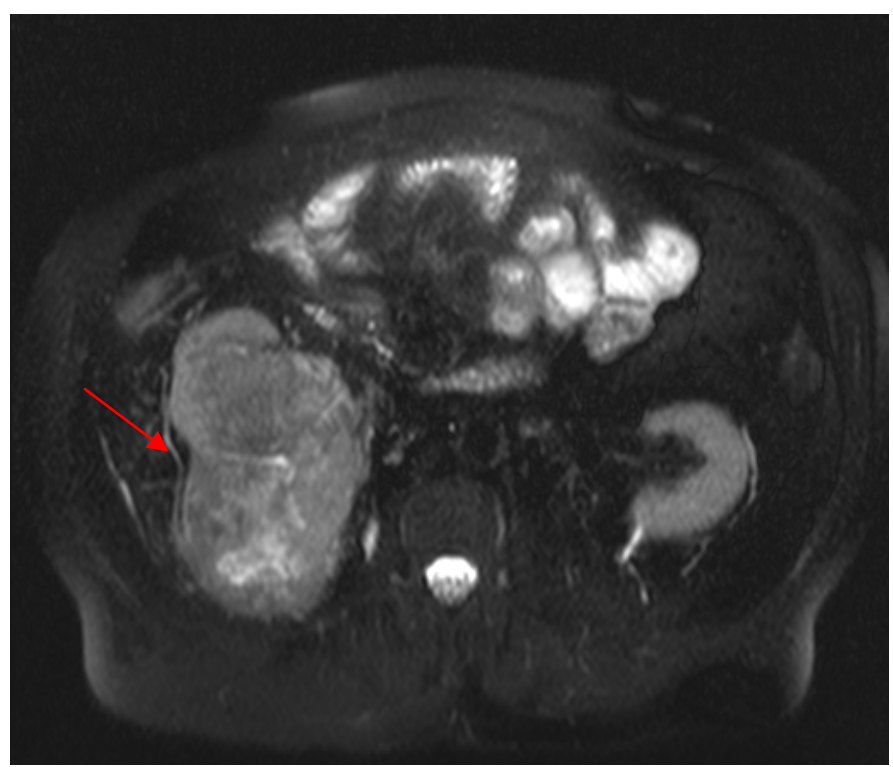

Figure 1. Large centrally necrotic mass in the right renal mid and lower pole which appears to extend into the right renal

Patient underwent a right radical cyto-reductive nephrectomy, biopsy of mesenteric mass, and left thoracotomy with biopsy in early 2003. Final pathology revealed grade 4 RCC, clear-cell type (Figure 2). There was tumor involvement into the perinephric fat and periureteral hilar soft tissue, and also tumor thrombus in the lumen of the renal vein. His left upper lobe lung biopsy was positive for vimentin, CAM5.2, and EMA.

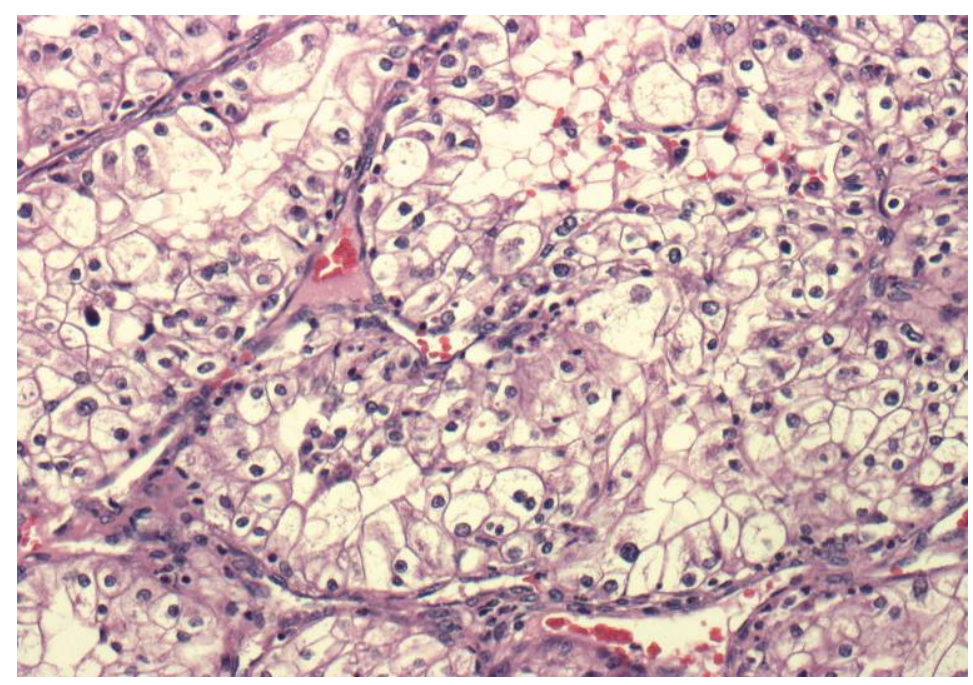

Figure 2. Clear cell renal cell carcinoma of kidney with typical clear cytoplasm and vascular pattern, 20X

Patient began treatment with Interferon Alpha $2 b-3$ million units subcutaneously three times a week. Patient tolerated regimen well and dose was increased to 5 million units three times a week. Two months later, CT scans showed an increase in the number and size of nodules in the lungs. Also, two new nodules were noted in the liver. Patient's therapy was switched to Interleukin-2 - 3 million units subcutaneously on days 1 through 5 . Secondary to Interleukin-2 therapy, patient experienced fever and chills (alleviated by acetaminophen), dry skin, and anemia for which he was placed on epoetin alfa.

CT scans demonstrated an increase in size of pulmonary nodules and the development of new bilateral nodules. Physical exam demonstrated a solid right axillary nodule. Biopsy was obtained; it revealed metastatic, poorly 
differentiated adenocarcinoma, clear cell type consistent with a renal primary. As third line treatment, patient was switched to bevacizumab $10 \mathrm{mg} / \mathrm{kg}$ IV every two weeks.

Following two months of treatment with bevacizumab, patient presented with anterior thigh pain. X-ray demonstrated a lytic lesion extending through the anterior cortex of the distal femoral. Palliation with radiation to this area was given. CT demonstrated a decrease in the size of masses in the lung. Bone scan revealed increased uptake in the distal femur and the presence of calvarial lesions. The dosing and frequency of bevacizumab had to be decreased because of proteinuria; zoledronic acid was added.

Patient presented a few weeks later for a lesion on his left back. During physical exam, a $2 \times 2.5 \mathrm{~cm}$ firm, immobile, subcutaneous nodule was noted (Figure 3). Pathological analysis revealed a tumor composed of pleomorphic and atypical epithelial cells with clear staining cytoplasm (Figure 4). Immunoperoxidase staining showed focal EMA positivity, keratin staining was negative, and vimentin staining was positive.

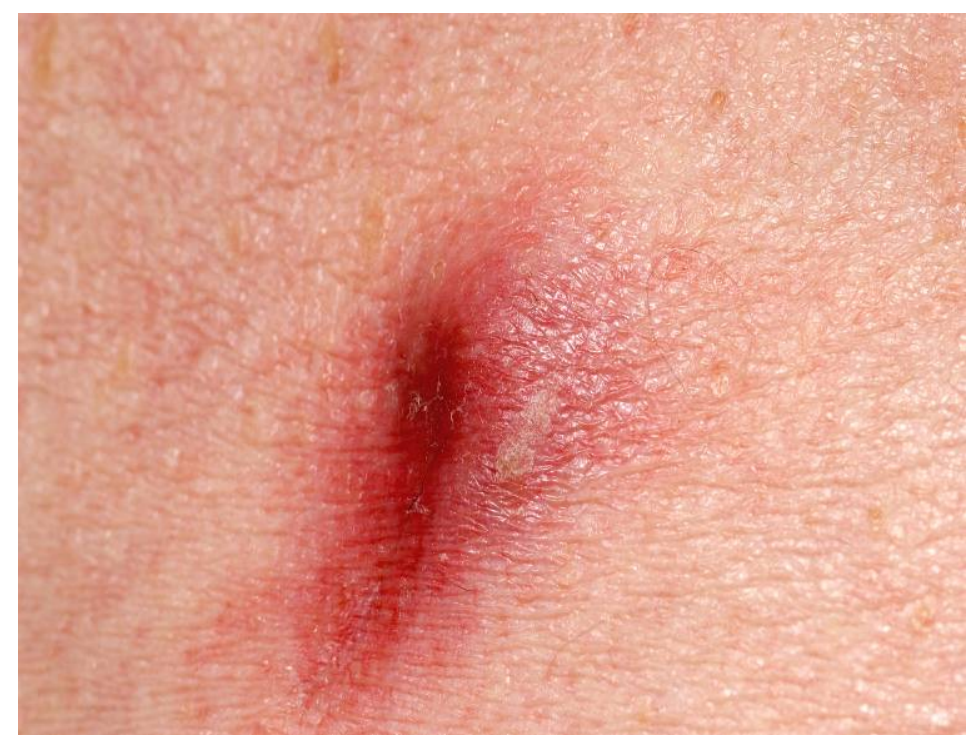

Figure 3.2 × $2.5 \mathrm{~cm}$ firm, immobile, subcutaneous nodule over left back

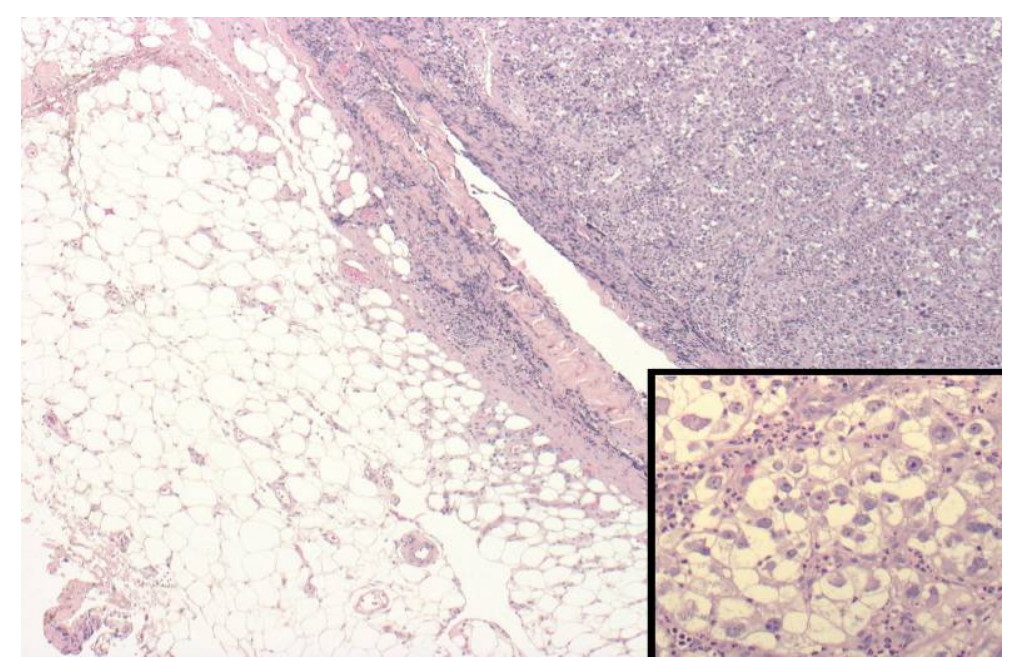

Figure 4. Metastatic clear cell carcinoma to the skin of the mid back, tumor infiltrates subcutaneous tissue

Thereafter, patient experienced diffuse bone pain. X-ray demonstrated a lucent lesion with cortical breakthrough in the left radius, and multiple lytic areas in the left femur. MRI of the brain revealed calvarial lesions with some break into the scalp soft tissue and into the epidural space. Bone scan and MRI were consistent with X-ray 
findings. New lesions of the skin on the scalp and right abdomen were noted (Figure 5). Because of response in visceral lesions, bevacizumab was resumed. Palliative radiation continued.

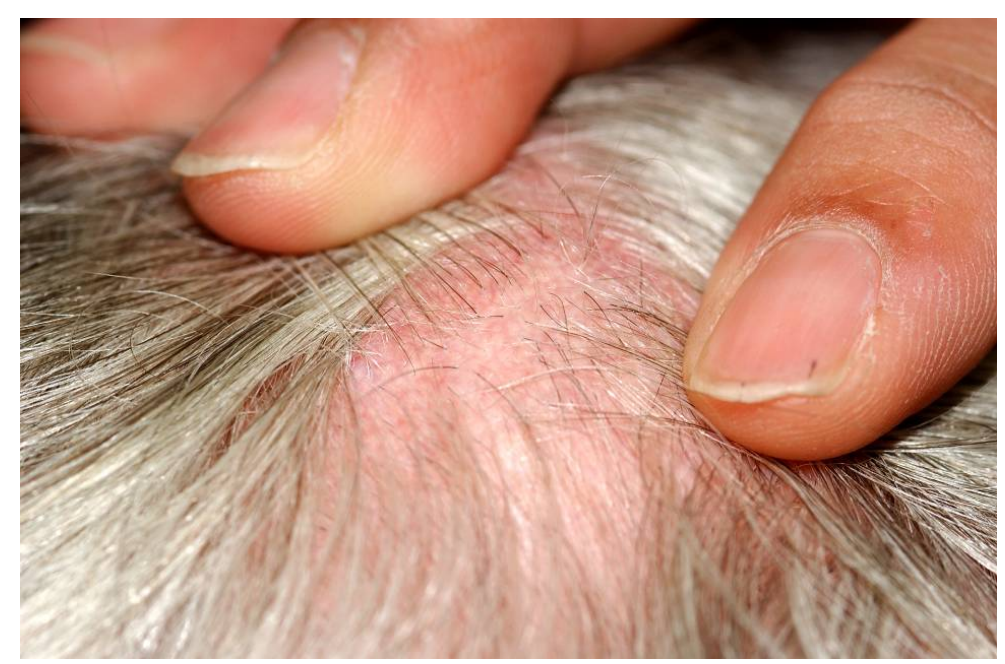

Figure 5. Subcutaneous lesion over scalp

CT showed evolving subcutaneous nodules in the right chest and anterior abdomen, new skin nodule in the right shoulder, and an enlarged lymph node in the right axilla. Erlotinib $150 \mathrm{mg}$ twice a day was added to bevacizumab to help control skin lesions. Erlotinib was reduced to $50 \mathrm{mg}$ following diarrhea and a pruritic skin rash.

A month later, erlotinib was stopped as patient developed headaches, nasal congestion, and nose bleeds. Physical exam revealed new nodules of the head. X-ray of facial bones demonstrated many poorly defined areas of osteolysis throughout the calvarium and base of skull.

Over the next 3 months, patient received radiation to skin lesions and bone as well as systemic treatment with bevacizumab. Patient's lung and subcutaneous nodules continued to grow and new lesions appeared. New lymph node involvement was noted on CT. Due to disease progression and low performance status, treatment was discontinued and patient was enrolled to hospice care.

\section{Discussion}

RCC comprises $80 \%$ of primary renal cancers in the USA (Jemal, 2006). Most cases are discovered incidentally during lab work and radiographical procedures. Most patients are asymptomatic upon diagnosis, yet $25 \%$ have advanced disease (Garnick, 1998). The most common locations for metastasis include brain, liver, local lymph nodes, bone, and lungs. Indeed, initial CT scan of this patient would reveal pulmonary nodules. Immunochemical analysis would be significant for a positive immune reaction to vimentin. Vimentin is an intermediate filament commonly found in mesoderm derived carcinomas such as RCC, CAM5.2, which reacts with secretory epithelia, but not stratified squamous epithelium, and EMA, a group of heavily glycosylated transmembrane proteins found in carcinomas rich in secretory epithelia. Overall, this immunochemical pattern would argue for metastatic RCC, and against a lung primary (Dhingra, 2011).

This patient's case was unique because it involved metastasis to the skin. RCC is the primary tumor in $6 \%$ of skin metastasis (Swartz, 2006). Few reports of recurrent RCC presenting with cutaneous metastases following nephrectomy exist. Most common sites reported are trunk than scalp (Porter, 2006; Koga, 2000). Skin metastasis from urological primaries generally presents as uticaria or a nonspecific macular-papular rash. In RCC, skin lesions have been described in the literature more specifically as pustules, painful and painless nodules, and macular entities. In a patient being treated with chemotherapy the differential of these manifestations is broad. It includes non-specific drug reactions, opportunistic skin infections, and also advanced metastatic disease. The definite diagnosis of metastasis is made through tissue biopsy. Patient's back nodule was found to be composed of pleomorphic and atypical epithelial cells with clear staining cytoplasm. Immunochemical analysis was consistent with RCC. 
Metastasis to the skin in RCC most often occurs via direct invasion of the skin overlying the tumor. Widespread metastasis is generally explained through hematological or lymphatic spread. The nodules on this patients scalp likely developed through neoplastic spread via arteriovenous and systemic shunts allowing tumor cells to bypass pulmonary filtration (Opper, 2006). Recent literature provides evidence that neoplastic cells may be implantated into the skin through diagnostic or surgical procedures (Nilufer, 2011), Cutaneous manifestations of urological tumors carry a poor prognosis as they are associated with widespread visceral involvement. Retrospective studies looking at urological malilgnancies that metastasized to the skin demonstrated a median survival time of 8 months (Thomas, 2004; Gleave, 1998).

Numerous modalities have been conceived to augment the anti-tumor response seen after nephrectomy. Interleukin-2 (IL-2) and interferon alfa (IFNa) have emerged as two of the most efficacious therapies. They likely enhance the ability of activated $\mathrm{T}$ cells and natural killer cells to destroy tumor cells.

Because of patient's history of myasthenia gravis and age, IFNa was chosen as the initial agent over IL-2 due to its limited toxicity. A retrospective study found five independent factors that predicted poorer survival with IFNa use; this patient had two: hemoglobin below the lower limit of normal and a period of less than 12 months from diagnosis to use (Motzer, 2002).

As therapy failed and given patient's co-morbid conditions, he was switched to low-dose IL-2 therapy. Patient experienced adverse reactions while on therapy, most serious being acute renal failure, noted in only $1 \%$ of patients (Yang, 1994). Patient remained on therapy for approximately ten months until CT demonstrated progressive disease. Furthermore, physical exam revealed an axillary nodule that became biopsy proven metastatic RCC.

Bevacizumab was chosen next due to its ability to inhibit vascular endothelial growth factor (VEGF) by binding to growth factor and decreasing microvascular growth and thus metastatic progression. Research initially involving von Hippel-Lindau (VHL) associated renal cell carcinomas found a loss of heterogeneity at the VHL locus on, a finding also present in 75 to 80 percent of sporadic RCCs (Maher, 1990). This loss of heterogeneity leads to an increased production of VEGF, which through tumor angiogenesis, plays a role in tumor growth and spread. A randomized, double- blind phase II clinical trial looking at patients who failed cytokine therapy found that, when compared to placebo, monotherapy with bevacizumab resulted in a significant increase in time of progression of disease. The probability of being disease free in 8 months while on high dose bevacizumab $(10 \mathrm{mg} / \mathrm{kg}$ ), like this patient, was suggested to be $30 \%$ (Yang, 2003).

As patient's disease continued to progress, the choice was made to add erlotinib, a tyrosine kinase inhibitor that blocks the intracellular binding of VEFG to its receptor. Early studies suggested increased survival time when compared to bevacizumab monotherpay. However, later research, including a phase II, randomized, double-blind, multicenter, placebo-controlled trial of 104 patients, would suggest that there is no difference in survival time between the two (Bukowski, 2007). This patient was treated in the era before sunitinib, pazopanib, temsirolimus, and sorafenib were approved (Table 1).

Table 1. Commonly used drugs in the treatment of rcc, their mechanisms of action, and major side effects

\begin{tabular}{lll}
\hline Drug & \multicolumn{1}{c}{ Mechanism of Action } & \multicolumn{1}{c}{ Major Side Effects } \\
\hline Interferon alfa-2B & $\begin{array}{l}\text { Through binding at specific membrane receptors on the cell surface, } \\
\text { it enhances the ability of activated T cells and natural killer cells to } \\
\text { destroy tumor cells. }\end{array}$ & $\begin{array}{l}\text { Fatigue, influenza-like symptoms, } \\
\text { Confusion, headache. skin reaction }\end{array}$ \\
Interleukin-2 & $\begin{array}{l}\text { Immunoregulatory properties including: enhancement of lymphocyte } \\
\text { toxicity and Induction of killer cell to destroy tumor cells }\end{array}$ & $\begin{array}{l}\text { Hypotension, diarrhea, oliguria. } \\
\text { skin reaction }\end{array}$ \\
Bevacizumab & $\begin{array}{l}\text { Inhibit vascular endothelial growth factor (VEFG) by binding to } \\
\text { growth factor and decreasing microvascular growth and thus } \\
\text { metastatic progression }\end{array}$ & $\begin{array}{l}\text { Hypertension, abdominal pain, } \\
\text { hemorrhage. skin reaction }\end{array}$ \\
& $\begin{array}{l}\text { Tyrosine kinase inhibitor that blocks the intracellular binding of } \\
\text { VEFG to its receptor }\end{array}$ & $\begin{array}{l}\text { Edema, infectious disease, fatigue, } \\
\text { abdominal pain skin reaction }\end{array}$ \\
\hline
\end{tabular}




\section{Conclusion}

This case helps highlight the ubiquity of RCC metastasis. This patient's disease would unfortunately progress to areas where RCC is commonly found, including the liver and lungs. However, his disease manifested rather uniquely, in the form of skin nodules. Our patient exhibited metastasis less than 2 years post nephrectomy, although it is generally seen years after surgery. As is typical, our patient suffered from synchronous visceral metastasis noted in $90 \%$ of cases Synchronous visceral metastasis were also noted in our patient, as it is typically in $90 \%$ of cases (Kouroupakis, 2008).

Furthermore, we have highlighted our treatment regimen for this patient and outlined our rationale for why it was chosen. Research continues to progress in terms of new modalities for suppressing and eliminating RCC. One of the more promising therapies to be developed is the use of drugs like temsirolimus and everolimus to decrease tumor burden by inhibiting an alterative pathway that is used for RCC angiogenesis and metastasis.

\section{References}

Bukowski, R. M., Kabbinavar, F. F., Figlin, R. A., Flaherty, K., Srinivas, S., Vaishampayan, U., ... McDermott, D. (2007). Randomized Phase II Study of Erlotinib Combined with Bevacizumab Compared with Bevacizumab Alone in Metastatic Renal Cell Cancer. $J$ Clin Oncol, 25(29), 4536-41. http://dx.doi.org/10.1200/JCO.2007.11.5154

Dhingra, V., Misra, V., Singh, A. P., \& Agarwal, S. (2011). Cytodiagnosis of cutaneous metastasis from renal cell carcinoma: A case report with review of literature. $J$ Cytol, 28, 30-2. http://dx.doi.org/10.4103/0970-9371.76947

Garnick, M. B. (1998). Primary neoplasms of the kidney. In: Therapy in Nephrology and Hypertension: A Companion to Brenner and Rector's the Kidney, Brady, HR, Wilcox, CS (Eds), WB Saunders.

Gleave, M. E., Elhilali, M., Fradet, Y., Davis, I., Venner, P., Saad, F., ... Bajamonde, A. N. (1998). Interferon gamma-1b compared with placebo in metastatic renal-cell carcinoma. C Engl J Med, 338(18), 1265. http://dx.doi.org/10.1056/NEJM199804303381804

Jemal, A., Siegeil, R., \& Xu, J. (2010). Cancer Statistics, 2010. CA Cancer J Clin, 60(5), 277-300. http://dx.doi.org/10.3322/caac.20073

Koga, S., Tsuda, S., Nishikido, M., Matsuya, F., Saito, Y., \& Kanetake, H. (2000). Renal cell carcinoma metastatic to the skin. Anticancer Res, 20, 1939-1940.

Kouroupakis, D., Patsea, E., Sofras, F., \& Apostolikas, N. (1995). Renal cell carcinoma metastases to the skin: a not so rare case? British Journal of Urology, 75, 583-585. http://dx.doi.org/10.1111/j.1464-410X.1995.tb07411.x

Maher, E. R., Yates, J. R., Harries, R., Benjamin, C., Harris, R., Moore, A. T., \& Ferguson-Smith, M. A. (1990). Clinical features and natural history of von Hippel-Lindau disease. NEJ Med, 77(283), 1151.

Motzer, R. J., Bacik, J., Murphy, B. A., Russo, P., \& Mazumdar, M. J. (2002). Interferon-alfa as a comparative treatment for clinical trials of new therapies against advanced renal cell carcinoma. Clin Oncol, 20(1), 289. http://dx.doi.org/10.1200/JCO.20.1.289

Mueller, T. J., Wu, H., Greenberg, R. E., Hudes, G., Topham, N., Lessin, S. R., \& Uzzo, R. G. (2004). Cutaneous metastases from genitourinary malignancies. Urology, 63(6), 1021-1026.

Onak Kandemir, N., Barut, F., Yılmaz, K., Tokgoz, H., Hosnuter, M., \& Ozdamar, S. O. (2010). Renal Cell Carcinoma Presenting with Cutaneous Metastasis: A Case Report. Case Report Med., 2010, 913734. http://dx.doi.org/10.1155/2010/913734

Opper, B., Elsner, P., \& Ziemer, M. (2006). Cutaneous metastasis of renal cell carcinoma. American Journal of Clinical Dermatology, 7(4), 271-272. http://dx.doi.org/10.2165/00128071-200607040-00010

Porter, N. A., Anderson, H. L., \& Al-Dujaily, S. (2006). Renal cell carcinoma presenting as a solitary cutaneous facial metastasis: case report and review of the literature. International Seminars in Surgical Oncology, 3, 27. http://dx.doi.org/10.1186/1477-7800-3-27

Swartz, M. A., Karth, J., Schneider, D. T., Rodriguez, R., Beckwith, J. B., \& Perlman, E. J. (2002). Renal medullary carcinoma: clinical, pathologic, immunohistochemical, and genetic analysis with pathogenetic implications. Urology, 60, 1083-1089. http://dx.doi.org/10.1016/S0090-4295(02)02154-4 
Yang, J. C., Haworth, L., Sherry, R. M., Hwu, P., Schwartzentruber, D. J., Topalian, S. L., ... Rosenberg, S. A. (2003). A randomized trial of bevacizumab, an anti-vascular endothelial growth factor antibody, for metastatic renal cancer. $N$ Engl J Med, 349(5), 427-34. http://dx.doi.org/10.1056/NEJMoa021491

Yang, J. C., Topalian, S. L., Parkinson, D., Schwartzentruber, D. J., Weber, J. S., Ettinghausen, S. E., ... Kim, H. I. (1994). Randomized comparison of high-dose and low-dose intravenous interleukin-2 for the therapy of metastatic renal cell carcinoma: an interim report. $J$ Clin Oncol, 12(8), 1572. http://dx.doi.org/10.1056/NEJMoa021491 